\title{
Effects of Bifidobacterium longum and Lactobacillus rhamnosus on Gut Microbiota in Patients with Lactose Intolerance and Persisting Functional Gastrointestinal Symptoms: A Randomised, Double-Blind, Cross-Over Study
}

\author{
Paola Vitellio ${ }^{1,2}$, Giuseppe Celano ${ }^{1}(0)$, Leonilde Bonfrate ${ }^{2}$, Marco Gobbetti ${ }^{3}$, \\ Piero Portincasa $2, t, *$ D and Maria De Angelis $1, t, * \mathbb{D}$ \\ 1 Department of Soil, Plant and Food Sciences, University of Bari Aldo Moro, via Amendola 165/a, \\ 70126 Bari, Italy; paolavitellio91@gmail.com (P.V.); g.celano1@gmail.com (G.C.) \\ 2 Clinica Medica "A. Murri", Department of Biomedical Sciences and Human Oncology, University of Bari \\ Medical School, 70121 Bari, Italy; leonildebnf@gmail.com \\ 3 Faculty of Science and Technology, Free University of Bolzano, piazza Università, 5, 39100 Bolzano, Italy; \\ marco.gobbetti@unibz.it \\ * Correspondence: piero.portincasa@uniba.it (P.P.); maria.deangelis@uniba.it (M.D.A.); \\ Tel.: +39-080-5478227 (P.P.); +39-080-5442949 (M.D.A.) \\ + These authors contributed equally to this work.
}

Received: 20 March 2019; Accepted: 16 April 2019; Published: 19 April 2019

check for updates

\begin{abstract}
Functional gastrointestinal symptoms are frequent, and may be driven by several pathogenic mechanisms. Symptoms may persist in lactose intolerant (LI) patients (i.e., subjects with intestinal lactase deficiency, lactose malabsorption producing symptoms), after a lactose-free diet. Our hypothesis was that probiotic and vitamin B6 treatment may be useful to alleviate symptoms in LI patients through a positive modulation of gut microbial composition and relative metabolism. We aimed to test the efficacy of a novel formulation of Bifidobacterium longum BB536 and Lactobacillus rhamnosus HN001 plus vitamin B6 (ZR) in 23 LI subjects with persistent symptoms during a lactose-free diet. Symptoms, microbiome, and metabolome were measured at baseline and after 30 days in a crossover, randomized, double-blind study of ZR versus placebo (PL). Compared with PL, the administration of probiotics and vitamin B6 significantly decreased bloating $(p=0.028)$ and ameliorated constipation $(p=0.045)$. Fecal microbiome differed between ZR and PL. ZR drove the enrichment of several genera involved in lactose digestion including Bifidobacerium. Moreover, the relative abundance of acetic acid, 2-methyl-propanoic acid, nonenal, and indolizine 3-methyl increased, while phenol decreased. Our findings highlight the importance of selected probiotics and vitamin B6 to alleviate symptoms and gut dysbiosis in lactose intolerant patients with persistent functional gastrointestinal symptoms.
\end{abstract}

Keywords: lactose intolerance; probiotics; vitamin B6; microbiome; metabolome

\section{Introduction}

Functional gastrointestinal diseases (FGIDs) are the most common cause of gastrointestinal disturbance in global population. Once organic (i.e., inflammatory or neoplastic) causes are ruled out, symptoms include a wide range of disorders [1] affecting the esophagus or upper gastrointestinal tract (i.e., functional dyspepsia, postprandial distress syndrome, or epigastric pain), bowel disorders (i.e., irritable bowel syndrome (IBS), constipation, diarrhea, or bloating), biliary and anorectal 
disorders, or dyspepsia. Several factors are involved in the genesis of FGIDs, and include intestinal dysbiosis [2,3], genetic predisposition, perception, diets, and intestinal dysmotility [4]. The exact physiopathology of FGIDs, however, remains poorly elucidated so far. FGIDs can also coexist with types of food intolerance. Lactose intolerance (LI) (due to lactase deficiency, down-regulation of lactase activity, lactose malabsorption causing abdominal symptoms upon lactose-containing products) [5-7] and fructose intolerance (due to fructose malabsorption with symptoms upon ingestion of fructose-containing foods) in adults [8] can easily mimic several FGIDs. Self-reported perception of LI can also affect the behaviour of patients in terms of diagnosis and therapeutic approaches [9-11]. However, even with a lactose- or fructose-restricted diet regimen, functional symptoms like constipation or diarrhoea, functional bloating, or dyspepsia may persist.

Recently, the idea of improving gut microbiota composition with some selected probiotic strains represents a valid therapeutical approach in FGIDs, even with concomitant LI [12]. Probiotics are defined as live bacteria or yeasts that positively supplement the gut microbiota [13]. Probiotic supplementation is a valid approach to maintain the balance of the intestinal microbiota, the composition of which could be altered by several factors (stress, lifestyle, diet, antibiotic therapies, and so on) [14]. Hence, probiotics can be administered for treatment of FGIDs, as well as carbohydrates malasorbition [15].

In particular, lactic acid bacteria (LAB) constitute a large part of intestinal microbiota and their probiotical properities are well documented [16]. The genus Lactobacillus is a source of interest in the prevention of some diseases including LI [17]. Moreover, the beneficial use of LAB and probiotics in general aids in the extraction of energy and nutrients, such as short-chain fatty acids (SCFAs) (acetate, propionate, butyrate) and amino acids from food [18]. Furthermore, the production of SCFAs stimulates ileal propulsive contractions, releases neuroendocrine factors, acidificates the intracolonic $\mathrm{pH}$, and relaxes intestinal gut tissues [19].

In addition, there is evidence that low intake of vitamin B6 might be associated with FGIDs such as IBS [20]. Notably, B6 is shown to have pleiotropic functions for human health [21].

The present study aimed to investigate the effects of a novel formulation of Bifidobacterium longum BB536 and Lactobacillus rhamnosus HN001 with vitamin B6 on symptoms, gut microbiota, and matabolome in a cohort of patients with persisting FGIDs on a lactose-free diet because of a prior diagnosis of LI.

\section{Materials and Methods}

\subsection{Patients and Study Design}

The initial group consisted of 135 adult symptomatic patients of both genders (54 males and 81 females). Figure 1 depicts the consort flow-chart of screened patients. Exclusion criteria were organic diseases with a diagnosis of structural abnormality of the gastrointestinal tract (i.e., inflammatory bowel diseases such as Crohn's disease, ulcerative colitis), pregnancy, abdominal surgery within the previous six months, infective diseases, drug or alcohol abuse, metabolic disturbances, mental illness, concomitant immunological, haematological or neoplastic disease, severe hepatic insufficiency (i.e., Child-Pugh class A-C), severe heart failure (NYHA class III-IV), and inability to provide the informed consent. Also excluded were 15 patients with a diagnosis of IBS according to the Rome IV criteria. IBS is characterized by recurrent chronic abdominal pain or discomfort and changes in stool or improvement with defecation, in the absence of detectable organic causes [22,23]. The 99 remaining patients underwent the hydrogen (H2) breath test to detect LI intolerance and 75 patients (19 males and 56 females, mean age $46 \pm 3.1$ SE years, range 20-67) were positive (Figure 1). The patients were encouraged to introduce lactose-free dairy products to undergo a lactose-free diet for at least six months. Patients $(N=34)$ were ultimately enrolled if symptoms (i.e., altered bowel habits such as constipation or diarrhea, bloating, and abdominal pain) persisted after six months. Because of 11 drop-out patients, the final cohort consisted of 23 subjects. 


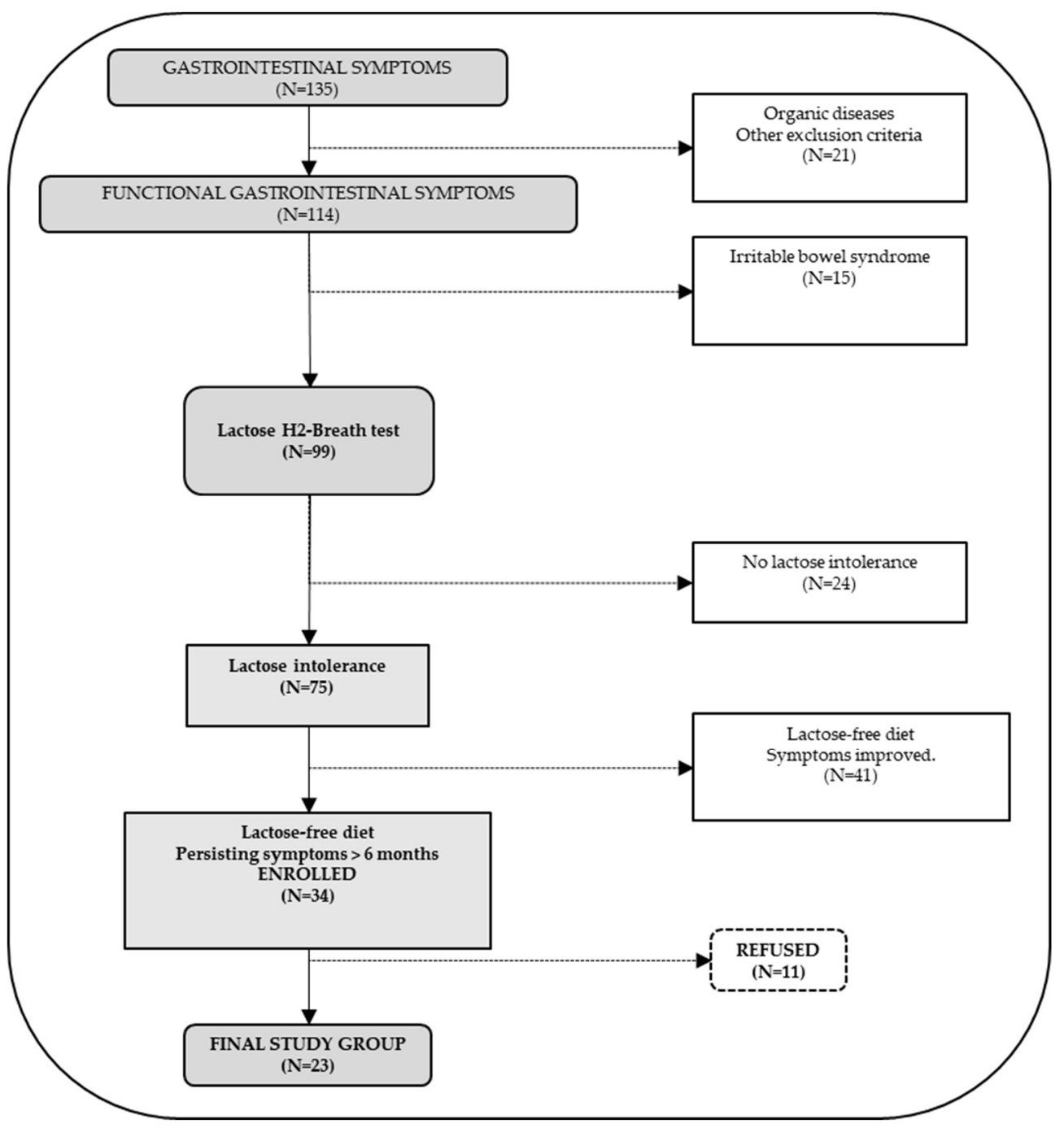

Figure 1. Consort flow-chart of screened patients. Starting from a general group of 135 symptomatic patients, and after exclusion of 21 patients with organic diseases, a subgroup of 114 patients had functional gastrointestinal symptoms. After further exclusions of 15 patients with Rome IV criteria, 99 patients underwent lactose $\mathrm{H} 2$ breath test and 75 were lactose intolerant, of which 34 had persisting symptoms following a lactose-free diet for at least six months, and were enrolled. Following the refusal of 11 subjects, the final study group consisted of 23 patients.

\subsection{Lactose Breath Test}

The lactose hydrogen breath test (LH2-BT) was performed according to previous guidelines [23] using lactose as fermentable substrate $(500 \mathrm{~mL}$ of cow's fresh whole milk containing $25 \mathrm{~g}$ of lactose). To avoid interferences with the fermentation process, a carbohydrate-free diet was prescribed the day before the test [24]. Smoking, physical exercise, and eating were not permitted $2 \mathrm{~h}$ before and during all test (180 $\mathrm{min})$ [25,26]. To measure the time-dependent concentrations of H2 in breath samples (as ppm), the operator used an automatic portable analyzer (Gastro + Gastrolyzer ${ }^{\circledR}$, Kent, England), with an accuracy of \pm 2 parts per million (ppm), a resolution of 1 ppm, and a range of 0-500 ppm. Subjects had to collect a breath sample across a mouthpiece connected to the Gastrolyzer at baseline and every 30 minutes. H2 levels were reported together with the presence of gastrointestinal symptoms (bloating, abdominal pain) and bowel movements [7]. The cut-off value for defining lactose malabsorption (absence of gastrointestinal symptoms) or LI (malabsorption and symptoms) was an $\mathrm{H} 2$ concentration 
of at least 20 ppm above the baseline (usually 1-2 ppm). Thus, only patients with LI (but not with simple lactose malabsorption or normal at $\mathrm{H} 2$ breath test) were enrolled.

\subsection{Randomization and Masking}

The study was a crossover, randomized, double-blind, placebo-controlled study (Figure 2), and was carried out at the Clinica Medica "A. Murri", Department of Biomedical Sciences \& Human Oncology, University of Bari Aldo Moro between January 2017 and December 2018. An independent researcher performed the randomization using a computer-generated randomization list. Alfasigma S.p.A. (Milano, Italy) provided the packets containing Zircombi ${ }^{\circledR}(\mathrm{ZR})$ and placebo (PL), and the randomization sequence for every patient according to the intervention treatments (ZR and PL). ZR and PL products were indistinguishable in appearance.

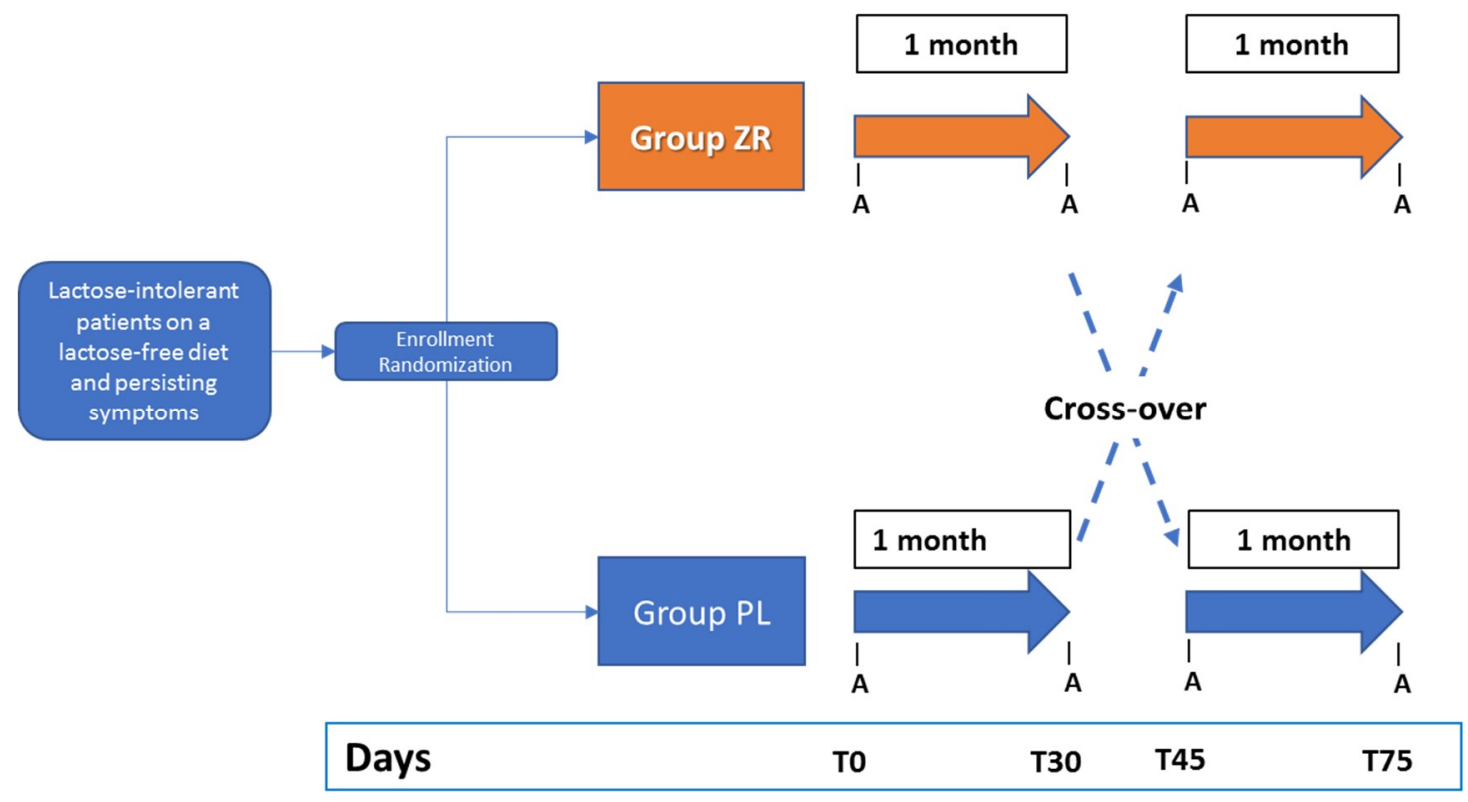

Figure 2. Crossover design of the study and timing of clinical evaluations. During the run-in (Ri) period, subjects fitting the inclusion criteria were randomized to receive either probiotic (ZR: $3 \mathrm{~g}$ as packets containing Bifidobacterium longum BB536 four billion CFU, Lactobacillus rhamnosus HN001 one billion CFU with B6 vitamin $1.4 \mathrm{mg}$ ) or placebo (PL: similar packets containing maltodextrins, corn starch, silicon dioxide, and no probiotic). The study included a first period of one month of treatment followed by 15 days of wash-out, and a second period of one month of treatment after cross-over. LI-lactose intolerant.

\subsection{Double Blind Cross Over Challenge}

During the run-in (Ri) period, subjects fitting the inclusion criteria were randomized to receive either probiotic (ZR: $3 \mathrm{~g}$ as packets containing B. longum BB536 four billion CFU, L. rhamnosus HN001 one billion CFU with B6 vitamin $1.4 \mathrm{mg}$ ) or placebo (PL: similar packets containing maltodextrins, corn starch, silicon dioxide, and no probiotic).

The study included a first period of one month of treatment followed by 15 days of wash-out, and a second period of one month of treatment after cross-over. Nonsteroidal anti-inflammatory drugs, anticoagulant, antibiotics, or probiotics were prohibited during the two weeks before baseline and throughout the study. Other treatments (i.e., antispasmodic, triptans, anticholinergics, motility regulating drugs and osmotic laxative, and antidepressant or anxiolytic drugs) were also prohibited at stable doses in the four weeks before randomization and during the whole study. Analyses were performed at days 0 (T0), 30 (T30), 45 (T45), and 75 (T75), and included symptoms, antrophometric evaluations, fecal microbiota, and related metabolome measurements. 
In order to assess compliance to study treatment and to record adverse events, patients were interviewed on a regular basis by medical personnel blinded to the regimen; compliance was calculated as the percentage of returned study product and a compliance was considered acceptable if $>80 \%$.

The study adhered to the Declaration of Helsinki and was approved by the Ethical Institutional Review Board of Institutional Ethics Committee of Bari University Hospital (study number 4651 ref. 11061CE, 02-10-2017). Written, informed consent was obtained from the patients, who were fully informed of the nature and purpose of the study. The study was registered in the Protocol Registration System Clinical Trial.gov (ClinicalTrials.gov Identifier: NCT03815617).

\subsection{Questionnaires}

Patients were evaluated for intensity of symptoms (abdominal pain and bloating, measured as visual analogue scale (VAS) in mm ranging from 0 to 100) and bowel movements were by the use of Bristol stool form scale (BSFS). Lifestyle and daily intake of foods were assessed using the MEDSTYLE questionnaire [8]. The adherence to a Mediterranean diet was calculated according to Sofi et al. [27].

\subsection{Outcomes}

The primary outcome was to determine whether dietary supplementation with ZL as compared with PL was able to improve symptoms (bloating and abdominal pain), as assayed by visual analogue scale (VAS). The scale is displayed as a horizontal line ranging from 0 to $100 \mathrm{~mm}$, where 0 is "no perception" and 100 is "the worst possible perception" [28].

The secondary outcome was the assessment of changes of bowel movements measured by the Bristol stool form scale. This scale represents a diagnosis instrument for classifying stool (1-7) and thus bowel habits. In particular, type 1-2 indicate constipation, type 3-4 indicate normal stool, and type 5-7 indicate diarrhoea [29]. A symptomatic response characterized by a decrease of at least $30 \%$ of the global VAS or Bristol stool form scale from the PL and ZR was defined as a clinical response.

\subsection{Fecal Collection}

Each subject provided two fecal samples at each study time point. After collection, feces were mixed with RNA later (Sigma-Aldrich, St. Louis, MO, USA) (ca. 5 g, 1:2 w/v) or with Amies transport medium (Oxoid LTD, Basingstoke, Hampshire, England) (ca. 15 g, 1:1 w/w), as previously described [30]. Fecal samples suspended in RNA later were stored at $-80{ }^{\circ} \mathrm{C}$ for RNA extraction. Samples diluted with Amies Transport were used for plate count (supplementary materials) and metabolome analysis.

\subsection{RNA Extraction and Fecal Microbiome}

Total genomic bacterial RNA was isolated from frozen stool samples using the Stool Total RNA purification KIT (Norgen Biotek Corp., Ontario, Canada), according to the manufacturer's instructions. The quality and concentration of the RNA were determined by spectrophotometric measurements at 260, 280, and $230 \mathrm{~nm}$ through the NanoDrop ND-1000 spectrophotometer. Total RNA extracted (approximately $2.5 \mu \mathrm{g}$ ) was retrotranscribed in cDNA using random hexamers and the Tetro cDNA synthesis kit (Bioline USA, Inc., Taunton, MA, USA), according to the manufacturer's instructions.

Bacterial Microbiome was estimated by $16 \mathrm{~S}$ rRNA. A $16 S$ metagenetic analysis was carried out at Genomix4life (spin-off of the University of Salerno, Fisciano, Italy) using the Illumina MiSeq platform. The V3-V4 region of the 16S rRNA gene was amplified for analysis of diversity inside the domains of bacteria [31]. PCR and sequencing analyses, quality control, and taxonomic assignment were carried out according to the protocol of Genomix4life. Shannon diversity and alpha diversity indices were calculated using Qiime analysis [32]. 


\subsection{Fecal Metabolome}

Fecal samples, placed into $10 \mathrm{~mL}$ glass vials, were sealed with polytetrafluoroethylene (PTFE)-coated silicone rubber septa, and then equilibrated for $10 \mathrm{~min}$ at $40{ }^{\circ} \mathrm{C}$. At the end of sample equilibration, a conditioned 50/30 $\mu \mathrm{m}$ DVB/CAR/PDMS fibre (Supelco, Bellefonte, PA, USA) was exposed to headspace for $40 \mathrm{~min}$ to extract volatile compounds by CombiPAL system injector autosampler (CTC Analytics, Zwingen, Switzerland). Volatile organic compounds (VOCs) were thermally desorbed by immediately transferring the fibre into the heated injection port $\left(220^{\circ} \mathrm{C}\right)$ of a Clarus 680 (Perkin Elmer, Beaconsfield UK) gas chromatography equipped with an Rtx-WAX column $(30 \mathrm{~m} \times 0.25 \mathrm{~mm}$ i.d., $0.25 \mu \mathrm{m}$ film thickness) (Restek) and coupled to a Clarus SQ8MS (Perkin Elmer) with source and transfer line temperatures kept at 250 and $210{ }^{\circ} \mathrm{C}$, respectively. The injection was carried out in splitless mode, and helium was used as the carrier gas at flow rate of $1 \mathrm{~mL} / \mathrm{min}$. The oven temperature was initially set at $35^{\circ} \mathrm{C}$ for $8 \mathrm{~min}$, then increased to $60{ }^{\circ} \mathrm{C}$ at $4{ }^{\circ} \mathrm{C} / \mathrm{min}$, to $160{ }^{\circ} \mathrm{C}$ at $6{ }^{\circ} \mathrm{C} / \mathrm{min}$, and finally to $200{ }^{\circ} \mathrm{C}$ at $20^{\circ} \mathrm{C} / \mathrm{min}$ and held for $15 \mathrm{~min}$. Electron ionization masses were recorded at $70 \mathrm{eV}$ in the mass-to-charge ratio interval, which was $\mathrm{m} / \mathrm{z} 34$ to 350 . The GC-MS generated a chromatogram with peaks representing individual compounds. Each chromatogram was analysed for peak identification using the National Institute of Standard and Technology 2008 (NIST) library. A peak area threshold of $>1000000$ and $85 \%$ or greater probability of match was used for VOC identification, followed by manual visual inspection of the fragment patterns when required. 4-Methyl-2-pentanol (final concentration $33 \mathrm{mg} / \mathrm{L}$ ) was used as an internal standard in all analyses, in order to quantify the identified compounds by interpolation of the relative areas versus the internal standard area.

\subsection{Statistical Analysis}

The sample size was calculated assuming a 35\% difference in response between treatment and placebo. We estimated that 20 patients would be required for the study to have $80 \%$ power and an $\alpha$ error of $5 \%$. A per-protocol analysis was applied to the trial. Normally distributed grouped data were expressed as the means and standard deviation $( \pm \mathrm{SD})$ and compared using paired and unpaired t-tests. Non-parametric grouped data were expressed as the means (95\% confidence interval (CI)) and compared using the Mann-Whitney rank sum test (paired) or Wilcoxon's signed rank test (unpaired). The randomization list was generated using the online resource available at www.randomization.com. Proportionate data were compared using Fisher's exact test or the $\chi^{2}$ test as appropriate. Differences between groups were analyzed using the two-tailed Student's t-test for independent samples. $p$-value $<0.05$ was regarded as significant [33]. The statistical software Statistica for Windows (Statistica 7.0) was used. Metagenomic and metabolomics data were subjected to permutation analysis using PermutMatrix and principal component analysis (PCA) using Statistica 7.0 for Windows.

\section{Results}

\subsection{Baseline Characteristics}

\subsubsection{Symptoms}

The final cohort consisted of 23 LI patients ( 4 males and 19 females). The baseline characteristics according to age, gender, body mass index (BMI), symptoms, and bowel habits appear in Table 1. Females constituted $83 \%$ of the study group. None of the subjects was obese, as testified by a mean of BMI of $23.2 \mathrm{Kg} / \mathrm{m}^{2}$ and median of $22.3 \mathrm{Kg} / \mathrm{m}^{2}$. With respect to symptoms, abdominal pain was of intermediate intensity; meanwhile, bloating had a higher score. The bowel habit analysis was in favour of patients suffering from constipation.

\subsubsection{Diet}

The amounts of daily micro/macronutrients at baseline appear in Table S1. Notably, patients followed a lactose-free diet for the six months prior to the enrollment and during the whole 
study period. Dietary fiber intake was lower than the recommended amount of daily fiber (adults $=12.6-16.7 \mathrm{~g} / 1000 \mathrm{kcal}$, SINU 2014). Also, the intake of daily carbohydrates $(41.64 \%$ of the total caloric intake) was lower than the recommended guidelines level ( $45 \%-60 \%$ daily calories intake, SINU 2014). In contrast, the total protein intake was within the reference range of $15 \%-20 \%$, with a prevalence of animal-type proteins versus plant protein. The total daily amount of lipids was higher than the recommended amount and represented $20 \%-35 \%$ of the total caloric intake. The percentage of saturated fatty acids was higher than the recommended by the guidelines for the prevention of cardiovascular diseases ( $<10 \%$ of the daily caloric intake, SINU 2014). The major source of monounsaturated fatty acids was olive oil, while the daily amount of vitamin B6 was $0.84 \pm 0.30 \mathrm{mg}$ per day, which was lower than the recommended daily average requirement $(1.1 \mathrm{mg} /$ day). The adherence score to Mediterranean diet for $96 \%$ patients was in the range of $10-15$ points (sufficiently adherent), while $4 \%$ of patients were included in the range of 5-9 points (low adherence).

Table 1. Baseline characteristics of the study patients $(N=23)$.

\begin{tabular}{cc}
\hline Age, years & $48 \pm 3.1(48)$ \\
\hline Females, $\boldsymbol{n}$. (\%) & $19(83 \%)$ \\
\hline BMI $\left(\mathbf{K g} / \mathbf{m}^{\mathbf{2}}\right)$ & $23.2 \pm 0.68(22.3)$ \\
\hline Symptoms & $69 \pm 5.4(80)$ \\
\hline Bloating (VAS, mm) & $61 \pm 4.6(62)$ \\
\hline Abdominal pain (VAS, mm) & \\
\hline Bowel habits & $3 \pm 0.38(2)$ \\
\hline
\end{tabular}

Legend: BMI, body mass index; VAS, visual analogue scale; data are mean \pm SEM (median).

\subsection{Clinical Scores during the Double Blind, Cross-Over Challenge}

The BMI remained stable throughout the observation period, as well as the intake of micro- and macronutrients. The symptom and bowel habits analysis according to sequence is reported in Table S2. The treatment with ZR caused a significant $(p=0.028)$ decrease of bloating, compared with placebo (PL) (Figure 3). No effect was evident in the case of abdominal pain (Figure S1).
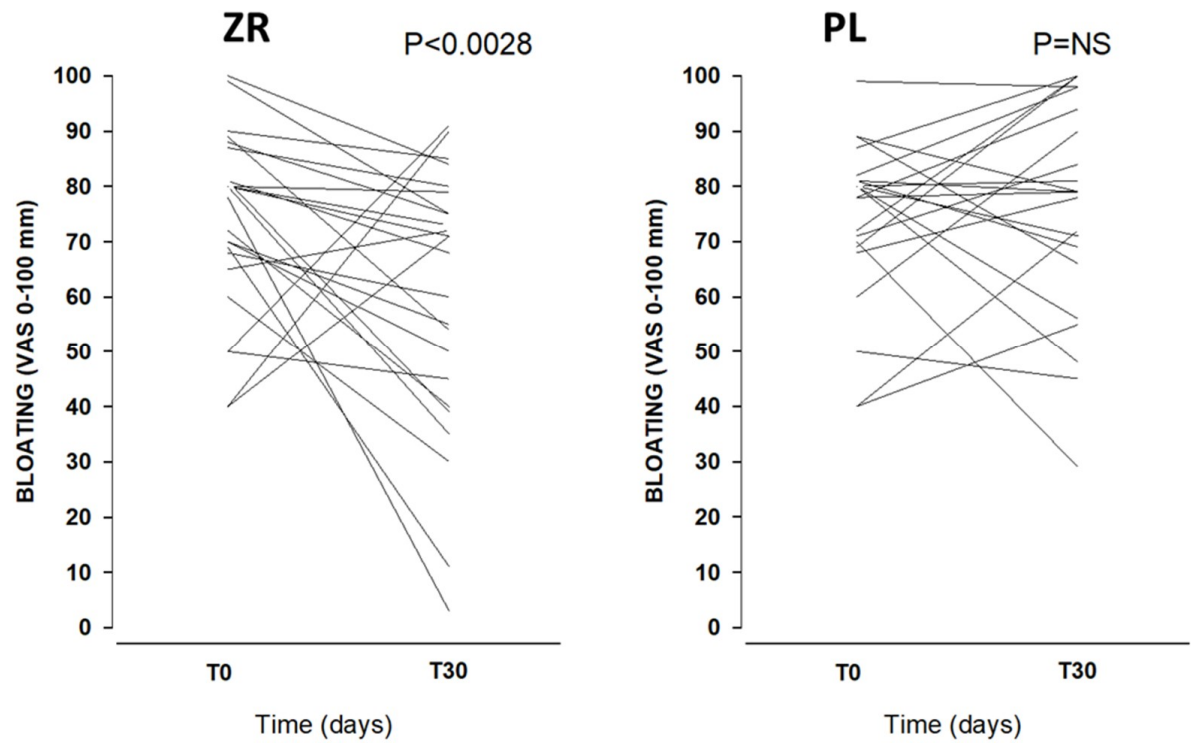

Figure 3. Representation of bloating (visual analogue scale (VAS) $0-100 \mathrm{~mm}$ ) in 23 patients at baseline (T0) and after 30 days (T30) of treatment (ZR) and placebo (PL) by spaghetti graft. 
The globally assessed Bristol score did not differ between placebo and ZR (Figure S2). However, at sub-analysis, the percent of patients with a score within the "normal" range of 3-4 increased from $30.4 \%$ to $73.9 \%$ after ZR ( $p=0.0072$, chi-squared). In particular, Bristol scores suggestive of constipation (i.e., $1-2)$ reached a normal range in $7 / 8$ patients $(87.5 \%)$ after $Z R$, while in patients with less formed stools (i.e., 5), 3/8 (37.5\%) scored normal after ZR ( $p=0.045)$. No statistically significant differences were found on the permeability data between placebo and $\mathrm{ZR}$.

\subsection{Probiotics and Vitamin B6 Affect the Fecal Microbiome of LI Patients}

A total number of 1,782,640 raw sequences referred to genus was obtained and analyzed; 1,573,358 reads passed the filters applied through the QIIME split_library.py script. The mean value of the percentage of reads classified to genus was $89 \%$. The average number of species operational taxonomic units (OTUs) identified in the fecal samples of ZR patients (mean value of 233) and PL (mean value of 179) significantly $(p=0.049)$ differed. Shannon index and $\beta$-diversity did not differ between ZL and PL samples (data not shown). Compared with 16S-rRNA (total bacteria), the highest significant differences between the fecal microbiota of the two groups (ZR and PL) were found using 16S-rRNA data (metabolically active bacteria). Eleven phyla (Firmicutes, Bacteroidetes, Proteobacteria, Actinobacteria, Chlorobi, Synergistetes, Spirochaetes, Tenericutes, Chloroflexi, Cyanobacteria, Fibrobacteres) were identified (Figure 4). Proteobacteria relative amount was higher $(p<0.05)$ in ZR samples compared with PL samples.
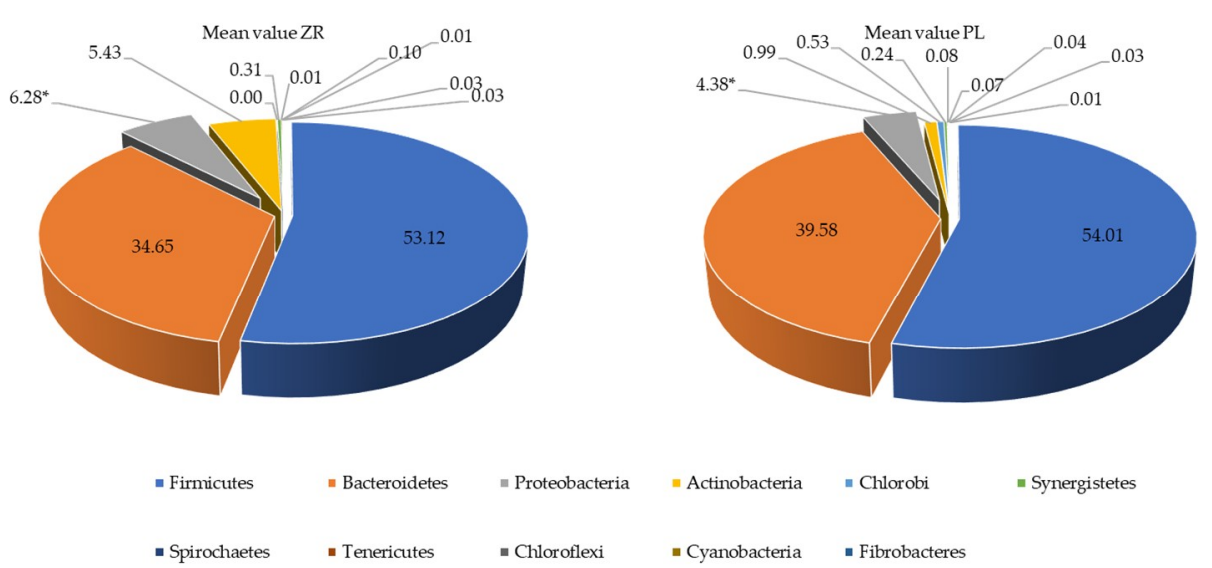

Figure 4. Relative proportions (percent) of phylum in the fecal samples of treated patients (ZR) and those of placebo (PL) patients.

The relative abundance of genus of Bifidobacterium was higher $(p=0.002)$ in $\mathrm{ZR}(0.46 \%)$ compared with PL group $(0.22 \%)$. Compared with PL, ZR drove several genus to a higher amount, including Slackia, Enterococcus, and Thricocccus ( $p<0.05)$. Lactobacillus genus did not differ between ZR and PL. A decreased relative abundance of Klesbiella, Serratia, and Enterobacter was detected in treated patients compared with PL ( $p<0.05$ ) (Figure 5). Compared to T0, the treatment ZR increased some components of the culturable microbiota (heterotrophic aerobic and anaerobic bacteria, presumptive lactic acid bacteria and Bifidobacterium) (Table S3). 


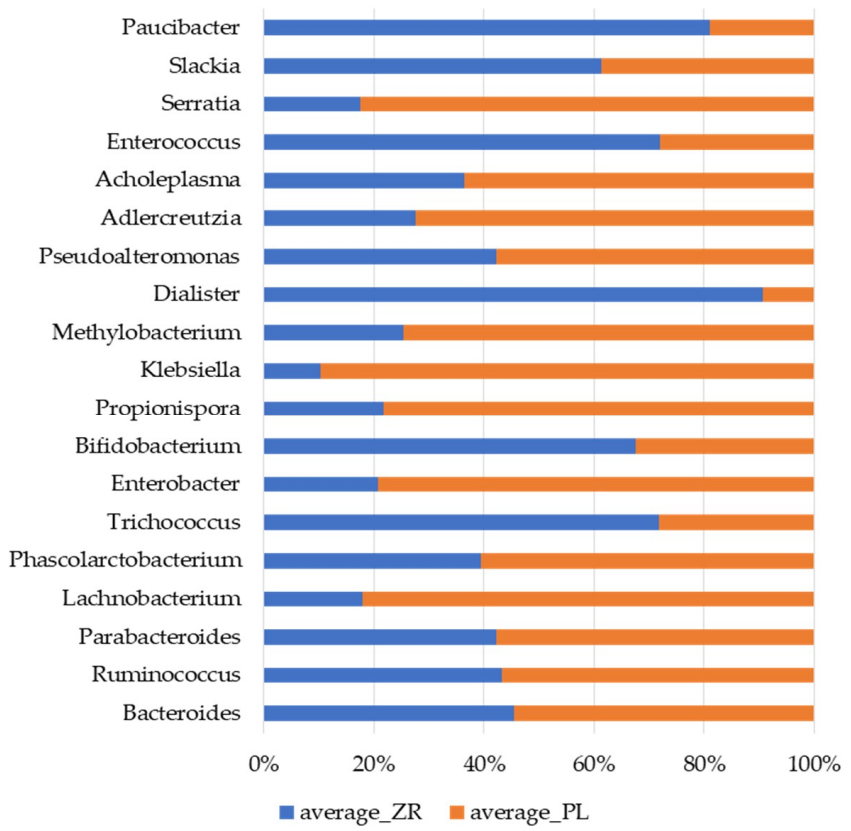

Figure 5. Relative proportions (percent) of genera showing significant $(p<0.05)$ differences between the fecal samples of treated patients (ZR) and those of placebo (PL) patients.

\subsection{Probiotics and Vitamin B6 Affect the Fecal Metabolome of LI Patients}

We investigated the differences within fecal volatile organic compounds (VOCs) in the ZR group compared with the PL group. VOCs identified from fecal samples (62 compounds) were grouped according to chemical classes, that is, alcohols (12), aldehydes (5), aromatic heterocyclic (2), hydrocarbons (8), ketones (8), short and medium chain fatty acids (SCFA) (10), sulfur compounds (2), esters and methyl esters (1), and terpens (14). The content of the metabolites within the same group largely varied; nevertheless, some significant differences were figured out between the two conditions (Table 2). Compared with PL, some compounds (acetic acid, 2-methyl-propanoic acid, nonenal, and indolizine 3-methyl) were found at significantly $(p<0.05)$ higher levels in the feces of ZR. Within significantly different alcohols $(p<0.05)$, alcohols 1-butanol, phenol, and 1-hexadecanol showed the highest level in the fecal samples of PL. Between the aldehydes compounds, hexanal was found at the highest levels in the feces samples of PL. Terpens were variously distributed within groups, except for limonene, which was significantly higher in the PL group.

Table 2. Volatile organic compounds (VOCs). Concentration (min, max) of VOCs (ppm) headspace fecal samples of treated (ZR) and placebo (PL) patients.

\begin{tabular}{cccc}
\hline Compounds & ZR & PL & $p$-value \\
\hline 1-Butanol & $0(0,0)$ & $11.22(0,12.58)$ & 0.037 \\
\hline 5-Hepten-2-ol, 6-methyl- & $51.74(5.98,72.3)$ & $30.81(0.79,39.28)$ & 0.029 \\
\hline Phenol & $4.12(0,8.56)$ & $19.78(3.48,28.56)$ & 0.048 \\
\hline 1-Hexadecanol & $4.03(0,6.29)$ & $8.17(0,13.94)$ & 0.043 \\
\hline Acetic Acid & $44.53(26.62,63.82)$ & $23.34(7.72,37.47)$ & 0.042 \\
\hline Propanoic Acid, 2-methyl- & $29.78(13.4,45.04)$ & $11.26(0.71,17.35)$ & 0.009 \\
\hline Hexanal & $5.81(0,13.22)$ & $11.3(2,19.38)$ & 0.009 \\
\hline 2-Nonenal & $5.38(2.24,8.9)$ & $3.35(0,4.34)$ & 0.045 \\
\hline Indolizine, 3-methyl- & $248.96(87.94,372.62)$ & $99.53(31,93.91)$ & 0.045 \\
\hline 5-Hepten-2-One, 6-methyl- & $310.76(55.31,396.75)$ & $124.03(19.91,210.84)$ & 0.048 \\
\hline D-Limonene & $87.43(11.19,187.9)$ & $483.68(55.46,804.44)$ & 0.042 \\
\hline
\end{tabular}




\subsection{Correlations between Microbiome and Metabolome}

Several correlations between diet, fecal microbiome, and metabolome existed (Figure 6). In particular, Bifidobacterium, Stachia, and Dialister genera were positively $(\mathrm{R}>0.700, \mathrm{FDR}<0.01)$ correlated with acetic acid, 2-methyl-propanoic acid, D-limonene, and 2-nonanal. Lactobacillus genus was positively correlated with acetic acid $(\mathrm{R}=0.667, \mathrm{FDR}=0.011)$. Bifidobacterium and Lactobacillus genera were negatively correlated with phenol $(\mathrm{R}<-0.650, \mathrm{FDR}<0.008)$. Interestingly, the relative abundance of fecal Bifidobacterium was also negatively correlated with bloating and abdominal pain of patients. On the contrary, Enterobacter and Serratia were correlated negatively $(\mathrm{R}<-0.700, \mathrm{FDR}<0.02)$ with acetic acid, 2-methyl-propanoic acid, D-limonene, and 2-nonanal, and positively associated $(\mathrm{R}>0.690, \mathrm{FDR}<0.03)$ with 1-butanol and phenol concentrations.

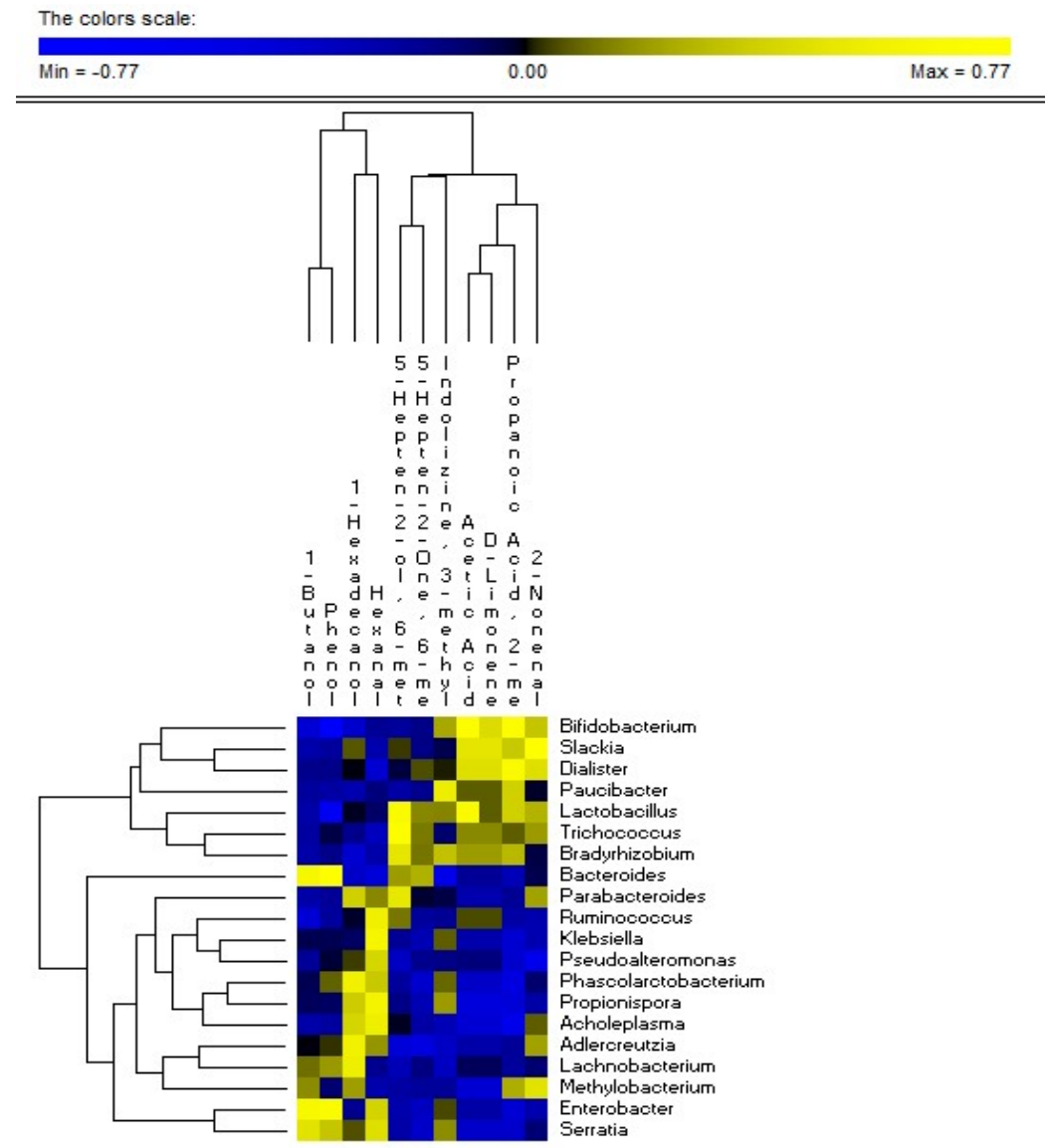

Figure 6. Significant correlations found between fecal microbiome and metabolome of lactose intolerant patients.

\section{Discussion}

In this study, we investigated common functional gastrointestinal symptoms, namely, abdominal pain, bloating, and bowel habits, in relation to intestinal permeability and fecal microbiota in patients treated with a specific probiotic containing B. longum BB536 and L. rhamnosus HN001 plus vitamin B6. In this cross-over, double blind, placebo-controlled, short-term study, we found consistent amelioration of some gastrointestinal symptoms, intestinal microbiota, and related metabolism with ZR, compared with placebo.

Also, as LI affects approximately 75\% of the world population [34], we enrolled the cohort with an established diagnosis of lactose intolerance and persisting symptoms after lactose-free diet for at least six months. We cannot exclude that persisting symptoms could originate from other dairy compounds, namely fats and proteins [35]. Indeed, fats in some dairy products were more likely to cause symptoms 
in at least two studies [36,37]. In addition, we cannot exclude that beta-casein proteins also activate gastrointestinal $\mu$-receptors, which, in turn, stimulate motility and abdominal symptoms [38,39]. Strain-specific bacterial metabolism might operate in the selection of probiotics and symbiotics to decrease the symptoms of gastrointestinal disorders, including LI [34]. Indeed, previous studies demonstrated that B. longum, as well as L. rhamnosus, improves general gastrointestinal symptoms, promotes lactose tolerance, and encourages a positive shift in gut microbial composition [40,41].

Bloating was most effectively corrected by ZR, according to the work of Zhu et al. [42]. Even with the lower intake of fibers than the recommended guidelines, constipation showed a trend towards improvement. Similar results have been previously described by Riezzo et al. [43]. Our data showed that the administration of B. longum BB536 and L. rhamnosus HN001 plus vitamin B6 for one month did not improve the intestinal permeability and abdominal pain. However, Yüce et al. [44] demonstrated that abdominal pain covers an important slice, but not the totality of LI patients with FGIDs. Recent research [45] suggests that probiotic bacteria formulation containing L. acidophilus, L. rhamnosus, L. casei, Bifidobacterium breve, S. thermophilus, B. longum, and B. infantis ameliorate flatulence and bloating in lactose malaborber patients. Our study suggests that similar results also stand for our patients.

Thirty days of treatment with B. longum BB536 and L. rhamnosus HN001 led to a positive shift in intestinal microbial composition. This modulation could be also the result of the intake of vitamin B6, according to the role of this vitamin in the metabolic pathways of microbes [46]. ZR drove an enrichment of genus Bifidobacterium. It is associated with lactose tolerance [47] and has been demonstrated to show relief in LI subjects with persistent FGIDs [41].

Recently, it was shown that short-chain galactooligosaccharide (GOS) induced a shift in the microbiome, increasing the relative amount of healthy promoting bacteria (Lactobacillus, Bifidobacterium, Faecalibacterium, and Roseburia), and a lowering of potentially detrimental species (Enterobacteriaceae) in LI patients [48]. In the present study, we found that the ratios of Lactobacillus-Bifidobacterium and Bacteroides-Enterobacteriaceae were respectively higher in ZR compared with those in PL. A lower ratio was demonstrated to be associated with food intolerance and allergy [49]. According to previous findings [48], Bifidobacterium showed a negative correlation with bloating and abdominal pain. Interestingly, the relative amount of some Bacteroides species was higher in PL compared with ZR. Some species of Bacteroides showed a pro-inflammatory effect [50]. In addition, a different balance of Lactobacillus species was found in ZR compared with PL. Lactobacillus species are known to be involved in LI relief [51]. However, other species have also been demonstrated to be involved in lactose digestion. Indeed, Phillips et al. [52] demonstrated that Escherichia spp. are involved in lactose breakdown.

The beneficial role in human gut health of acetic, propionic, and butanoic acids, against different types of disease, has already been well described [52,53]. Short chain fatty acids (SCFAs) might contribute to responses of immune cells in diseases associated with alterations in populations of commensal bacteria (dysbiosis) [54,55]. Fibers or dietary plant polysaccharides, such as non-digested oligosaccharides and proteins, are fermented by the microbiota populations to produce SCFAs [56]. In particular, Bifidobacterium, belonging to the phylum Actinobacteria and the groups of lactobacilli, are the main bacteria playing a central role in SCFAs' metabolism [57]. The higher level of acetic acid and 2-methyl-propanoic acid during the treatment could be related to the increase of Bifidobacteirum [58]. Accordingly, the amount Bifidobacterium was positively correlated with acetic acid and 2-methyl-propanoic acid. Acetate is an important SCFA present in the colon, which could have a trophic effect on the colonic epithelium not only by local action, but also by raising the mucosal blood flux [59]. In contrast to SFCAs, the products of amino acid fermentation impair colon health, and so their presence is undesirable. Bifidobacterium and Lactobacillus genera negatively correlated with the concentration of phenol in fecal samples. Clostridiaceae are one of the main bacterial groups, which synthesizes some metabolic products (e.g., phenols, p-cresol, certain indole derivatives) that are potentially toxic for humans $[30,60]$. Positive correlations were found between the relative amount of Proteobacteria genera (Serratia and Enterobacter) and the concentration of phenol in fecal samples of LI 
patients. A significant decrease of fecal phenol observed in ZR compared with PL could be explained by considering the Bifidobacterium shift in gut microbial population.

\section{Conclusions}

According to our results, probiotic and vitamin B6 treatment may be useful to alleviate symptoms in subjects with LI and persistent FGIDs through a positive modulation of gut microbial composition and relative metabolism. One trial limitation is the relatively short duration of treatment. The randomized, cross-over design of the protocol, however, partly overcomes this problem, although longer studies need to be addressed in the near future.

Supplementary Materials: The following are available online at: http://www.mdpi.com/2072-6643/11/4/886/s1. Figure S1: Representation of abdominal pain (VAS 0-100 nm) in 23 patients at baseline (T0) and after 30 days (T30) of treatment (ZR) and placebo (PL) by spaghetti graft. Figure S2: Representation of Bristol Score (1-7) at baseline (T0) in 23 patients and after 30 days (T30) of treatment (ZR) and placebo (PL) by spaghetti graft. Table S1: Amounts of daily micro/macronutrients at baseline. Table S2: Changes of gastrointestinal symptoms and bowel habits in placebo (PL) and treatment $(\mathrm{ZR})$ according to sequence $(1=\mathrm{Placebo} / \mathrm{ZR}$ and $2=\mathrm{ZR} / \mathrm{Placebo})$ and globally. Table S3: Median values and range of cultivable of bacterial cells $(\log \mathrm{CFU} / \mathrm{g}$ ) of the main microbial groups in the faecal samples of lactose intolerant patients at baseline (T0), after 30 days of treatment (ZR) or Placebo (PL).

Author Contributions: Conceptualization, P.P.; methodology, P.P., M.G., M.D.A. and L.B.; formal analysis, P.V., G.C.; investigation, P.V., G.C., L.B., and M.D.A.; data curation, M.D.A., P.V., and G.C.; writing-original draft preparation, P.V.; writing-review and editing, P.P. and M.D.A.; supervision, P.P. and M.D.A.; project administration, M.D.A. and P.P.; funding acquisition, M.D.A. and P.P.

Funding: This research was funded by Programma Operativo Nazionale, Ricerca e Innovazione 2014-2020, PON-FSE-FESR RESEARCH AND INNOVATION 2014-2020 DR n. 993 30.03.2017.

Acknowledgments: We thank Domenica Di Palo for helpful enrollment of patients and sample collection.

Conflicts of Interest: The authors declare no conflict of interest. The funders had no role in the design of the study; in the collection, analyses, or interpretation of data; in the writing of the manuscript; or in the decision to publish the results.

\section{References}

1. Drossman, D.A. Functional Gastrointestinal Disorders: History, pathophysiology, clinical features and Rome IV. Gastroenterology 2016, 150, 1262-1279. [CrossRef] [PubMed]

2. De Palma, G.; Collins, S.M.; Bercik, P. The microbiota-gut-brain axis in functional gastrointestinal disorders. Gut. Microb. 2014, 5, 419-429. [CrossRef] [PubMed]

3. Bonfrate, L.; Tack, J.; Grattagliano, I.; Cuomo, R.; Portincasa, P. Microbiota in health and irritable bowel syndrome: Current knowledge, perspectives and therapeutic options. Scand. J. Gastroenterol. 2013, 48, 995-1009. [CrossRef] [PubMed]

4. Portincasa, P.; Moschetta, A.; Baldassarre, G.; Altomare, D.F.; Palasciano, G. Pan-enteric dysmotility, impaired quality of life and alexithymia in a large group of patients meeting ROME II criteria for irritable bowel syndrome. World J. Gastroenterol. 2003, 9, 2293-2299. [CrossRef]

5. Liebert, A.; Lopez, S.; Jones, B.L.; Montalva, N.; Gerbault, P.; Lau, W.; Thomas, M.G.; Bradman, N.; Maniatis, N.; Swallow, D.M. World-wide distributions of lactase persistence alleles and the complex effects of recombination and selection. Hum. Genet. 2017, 136, 1445-1453. [CrossRef] [PubMed]

6. Wilson, K.; Hill, R.J. The role of food intolerance in functional gastrointestinal disorders in children. Aust. Fam. Phys. 2014, 43, 686-689.

7. Portincasa, P.; Di Ciaula, A.; Vacca, M.; Montelli, R.; Wang, D.Q.; Palasciano, G. Beneficial effects of oral tilactase on patients with hypolactasia. Eur. J. Clin. Investig. 2008, 38, 835-844. [CrossRef] [PubMed]

8. Bonfrate, L.; Krawczyk, M.; Lembo, A.; Grattagliano, I.; Lammert, F.; Portincasa, P. Effects of dietary education, followed by a tailored fructose-restricted diet in adults with fructose malabsorption. Eur. J. Gastroenterol. Hepatol. 2015, 27, 785-796. [CrossRef] [PubMed]

9. Yang, J.; Deng, Y.; Chu, H.; Cong, Y.; Zhao, J.; Pohl, D.; Misselwitz, B.; Fried, M.; Dai, N.; Fox, M. Prevalence and presentation of lactose intolerance and effects on dairy product intake in healthy subjects and patients with irritable bowel syndrome. Clin. Gastroenterol. Hepatol. 2013, 11, 262-268. [CrossRef] [PubMed] 
10. Barr, S.I. Perceived lactose intolerance in adult Canadians: A national survey. Appl. Physiol. Nutr. Metab. 2013, 38, 830-835. [CrossRef]

11. Farup, P.G.; Monsbakken, K.W.; Vandvik, P.O. Lactose malabsorption in a population with irritable bowel syndrome: Prevalence and symptoms. A case-control study. Scand. J. Gastroenterol. 2004, 39, 645-649. [CrossRef]

12. The effects of probiotics in lactose intolerance: A systematic review. Crit. Rev. Food Sci. Nutr. 2018. [CrossRef]

13. Tomasik, P.J.; Tomasik, P. Probiotics and prebiotics. Cereal Chem. 2003, 80, 113-117. [CrossRef]

14. Hemarajata, P.; Versalovic, J. Effects of probiotics on gut microbiota: Mechanisms of intestinal immunomodulation and neuromodulation. Ther. Adv. Gastroenterol. 2013, 6, 39-51. [CrossRef] [PubMed]

15. Harper, A.; Naghibi, M.M.; Garcha, D. The role of bacteria, probiotics and diet in Irritable Bowel Syndrome. Foods 2018, 7, 13. [CrossRef]

16. Wang, Y.; Wu, Y.; Wang, Y.; Xu, H.; Mei, X.; Yu, D.; Wang, Y.; Li, W. Antioxidant properties of probiotic bacteria. Nutrients 2017, 9, 521. [CrossRef]

17. Honda, H.; Kataoka, F.; Nagaoka, S.; Kawai, Y.; Kitazawa, H.; Itoh, H.; Kimura, K.; Taketomo, N.; Yamazaki, Y.; Tateno, Y.; Saito, T. Beta-galactosidase, phospho-beta-galactosidase and phospho-beta-glucosidase activities in lactobacilli strains isolated from human faeces. Lett. Appl. Microbiol. 2007, 45, 461-466. [CrossRef]

18. Den Besten, G.; van Eunen, K.; Groen, A.K.; Venema, K.; Reijngoud, D.-J.; Bakker, B.M. The role of short-chain fatty acids in the interplay between diet, gut microbiota, and host energy metabolism. J. Lipid Res. 2013, 54, 2325-2340. [CrossRef]

19. Hurst, N.R.; Kendig, D.M.; Murthy, K.S.; Grider, J.R. The short chain fatty acids, butyrate and propionate, have differential effects on the motility of the guinea pig colon. Neurogastroenterol. Motil. 2014, 26, 1586-1596. [CrossRef] [PubMed]

20. Ligaarden, S.C.; Farup, P.G. Low intake of vitamin B6 is associated with irritable bowel syndrome symptoms. Nutr. Res. 2011, 31, 356-361. [CrossRef] [PubMed]

21. Hellmann, H.; Mooney, S. Vitamin B6: A molecule for human health? Molecules 2010, 15, 442-459. [CrossRef]

22. Mearin, F.; Lacy, B.E.; Chang, L.; Chey, W.D.; Lembo, A.J.; Simren, M.; Spiller, R. Bowel Disorders. Gastroenterology 2016. [CrossRef]

23. Gasbarrini, A.; Corazza, G.R.; Gasbarrini, G.; Montalto, M.; Di Stefano, M.; Basilisco, G.; Parodi, A.; Usai-Satta, P.; Vernia, P.; Anania, C.; et al. Methodology and indications of H2-breath testing in gastrointestinal diseases: The Rome Consensus Conference. Aliment. Pharmacol. Ther. 2009, 29, 1-49. [PubMed]

24. Di Stefano, M.; Missanelli, A.; Miceli, E.; Strocchi, A.; Corazza, G.R. Hydrogen breath test in the diagnosis of lactose malabsorption: Accuracy of new versus conventional criteria. J. Lab. Clin. Med. 2004, 144, 313-318. [CrossRef]

25. Thompson, D.G.; O'Brien, J.D.; Hardie, J.M. Influence of the oropharyngeal microflora on the measurement of exhaled breath hydrogen. Gastroenterology 1986, 91, 853-860. [CrossRef]

26. Payne, D.L.; Welsh, J.D.; Claypool, P.L. Breath hydrogen (H2) response to carbohydrate malabsorption after exercise. J. Lab. Clin. Med. 1983, 102, 147-150.

27. Sofi, F.; Abbate, R.; Gensini, G.F.; Casini, A. Accruing evidence on benefits of adherence to the Mediterranean diet on health: An updated systematic review and meta-analysis. Am. J. Clin. Nutr. 2010, 92, 1189-1196. [CrossRef] [PubMed]

28. Bonfrate, L.; Scaccianoce, G.; Palasciano, G.; Ben-Chetrit, E.; Portincasa, P. A novel cluster of patients with Familial Mediterranean Fever (FMF) in southern Italy (J COVER). Eur. J. Clin. Investig. 2017, 47, 622-629. [CrossRef] [PubMed]

29. Lewis, S.J.; Heaton, K.W. Stool form scale as a useful guide to intestinal transit time. Scand. J. Gastroenterol. 1997, 32, 920-924. [CrossRef]

30. De Angelis, M.; Piccolo, M.; Vannini, L.; Siragusa, S.; De Giacomo, A.; Serrazzanetti, D.I.; Cristofori, F.; Guerzoni, M.E.; Gobbetti, M.; Francavilla, R. Fecal microbiota and metabolome of children with autism and pervasive developmental disorder not otherwise specified. PLOS ONE 2013, 8, e76993. [CrossRef] [PubMed]

31. Klindworth, A.; Pruesse, E.; Schweer, T.; Peplies, J.; Quast, C.; Horn, M.; Glockner, F.O. Evaluation of general $16 \mathrm{~S}$ ribosomal RNA gene PCR primers for classical and next-generation sequencing-based diversity studies. Nucleic Acids Res. 2013, 41, e1. [CrossRef]

32. Chao, A.; Bunge, J. Estimating the number of species in a stochastic abundance model. Biometrics 2002, 58, 531-539. [CrossRef] 
33. Tallarida, R.J.; Murray, R.B. Manual of Pharmacologic Calculations with Computer Programs; Springer: New York, NY, USA, 1987.

34. Arnold, J.; Simpson, J.; Roach, J.; Bruno-Barcena, J.; Azcarate-Peril, M. Prebiotics for Lactose Intolerance: Variability in galacto-oligosaccharide utilization by intestinal Lactobacillus rhamnosus. Nutrients 2018, 10, 1517. [CrossRef]

35. Szilagyi, A.; Ishayek, N. Lactose Intolerance, dairy avoidance, and treatment options. Nutrients 2018, 10, 1994. [CrossRef] [PubMed]

36. Nolan-Clark, D.; Tapsell, L.C.; Hu, R.; Han, D.Y.; Ferguson, L.R. Effects of dairy products on Crohn's Disease Symptoms are influenced by fat content and disease location but not lactose content or disease activity status in a New Zealand population. J. Am. Diet. Assoc. 2011, 111, 1165-1172. [CrossRef] [PubMed]

37. Mishkin, S. Dairy sensitivity, lactose malabsorption, and elimination diets in inflammatory bowel disease. Am. J. Clin. Nutr. 1997, 65, 564-567. [CrossRef] [PubMed]

38. Pal, S.; Woodford, K.; Kukuljan, S.; Ho, S. Milk Intolerance, beta-casein and lactose. Nutrients 2015, 7, 7285-7297. [CrossRef]

39. Jianqin, S.; Leiming, X.; Lu, X.; Yelland, G.W.; Ni, J.; Clarke, A.J. Effects of milk containing only A2 beta casein versus milk containing both $\mathrm{A} 1$ and $\mathrm{A} 2$ beta casein proteins on gastrointestinal physiology, symptoms of discomfort, and cognitive behavior of people with self-reported intolerance to traditional cows' milk. Nutr. J. 2015, 15, 35. [CrossRef] [PubMed]

40. Collado, M.C.; Gueimonde, M.; Sanz, Y.; Salminen, S. Adhesion properties and competitive pathogen exclusion ability of bifidobacteria with acquired acid resistance. J. Food Prot. 2006, 69, 1675-1679. [CrossRef]

41. Agustina, R.; Lukito, W.; Firmansyah, A.; Suhardjo, H.N.; Murniati, D.; Bindels, J. The effect of early nutritional supplementation with a mixture of probiotic, prebiotic, fiber and micronutrients in infants with acute diarrhea in Indonesia. Asia Pac. J. Clin. Nutr. 2007, 16, 435-442.

42. Zhu, Y.; Zheng, X.; Cong, Y.; Chu, H.; Fried, M.; Dai, N.; Fox, M. Bloating and distention in irritable bowel syndrome: The role of gas production and visceral sensation after lactose ingestion in a population with lactase deficiency. Am. J. Gastroenterol. 2013, 108, 1516-1525. [CrossRef] [PubMed]

43. Riezzo, G.; Orlando, A.; D’Attoma, B.; Linsalata, M.; Martulli, M.; Russo, F. Randomised double blind placebo controlled trial on Lactobacillus reuteri DSM 17938: Improvement in symptoms and bowel habit in functional constipation. Benef. Microbes 2018, 9, 51-60. [CrossRef] [PubMed]

44. Yuce, O.; Kalayci, A.G.; Comba, A.; Eren, E.; Caltepe, G. Lactose and Fructose Intolerance in Turkish children with Chronic Abdominal Pain. Ind. Pediatr. 2016, 53, 394-397. [CrossRef]

45. Use of a Novel Probiotic Formulation to Alleviate Lactose Intolerance Symptoms-A Pilot Study. Probiotics Antimicrob. Proteins 2019. [CrossRef]

46. Rosenberg, J.; Ischebeck, T.; Commichau, F.M. Vitamin B6 metabolism in microbes and approaches for fermentative production. Biotechnol. Adv. 2017, 35, 31-40. [CrossRef] [PubMed]

47. Arola, H.; Tamm, A. Metabolism of lactose in the human body. Scand. J. Gastroenterol. Suppl. 1994, 202, 21-25. [CrossRef] [PubMed]

48. Azcarate-Peril, M.A.; Ritter, A.J.; Savaiano, D.; Monteagudo-Mera, A.; Anderson, C.; Magness, S.T.; Klaenhammer, T.R. Impact of short-chain galactooligosaccharides on the gut microbiome of lactose-intolerant individuals. Proc. Natl. Acad. Sci. USA 2017, 114, E367-E375. [CrossRef] [PubMed]

49. De Angelis, M.; Vannini, L.; Di Cagno, R.; Cavallo, N.; Minervini, F.; Francavilla, R.; Ercolini, D.; Gobbetti, M. Salivary and fecal microbiota and metabolome of celiac children under gluten-free diet. Int. J. Food Microbiol. 2016, 239, 125-132. [CrossRef] [PubMed]

50. Setoyama, H.; Imaoka, A.; Ishikawa, H.; Umesaki, Y. Prevention of gut inflammation by Bifidobacterium in dextran sulfate-treated gnotobiotic mice associated with Bacteroides strains isolated from ulcerative colitis patients. Microbes Infect. 2003, 5, 115-122. [CrossRef]

51. Pakdaman, M.N.; Udani, J.K.; Molina, J.P.; Shahani, M. Erratum to: The effects of the DDS-1 strain of Lactobacillus on symptomatic relief for lactose intolerance-A randomized, double-blind, placebo-controlled, crossover clinical trial. Nutr. J. 2016, 15, 83. [CrossRef] [PubMed]

52. Vipperla, K.; O'Keefe, S.J. The microbiota and its metabolites in colonic mucosal health and cancer risk. Nutr. Clin. Pract. 2012, 27, 624-635. [CrossRef] 
53. Thorburn, A.W.; Storlien, L.H.; Jenkins, A.B.; Khouri, S.; Kraegen, E.W. Fructose-induced in vivo insulin resistance and elevated plasma triglyceride levels in rats. Am. J. Clin. Nutr. 1989, 49, 1155-1163. [CrossRef] [PubMed]

54. Portincasa, P.; Lembo, A.; De Bari, O.; Di Palo, D.M.; Maggio, A.; Cataldo, I.; Calamita, G. The role of dietary approach in irritable bowel syndrome. Curr. Med. Chem. 2017, 24, 1-8. [CrossRef]

55. Portincasa, P.; Bonfrate, L.; de Bari, O.; Lembo, A.; Ballou, S. Irritable bowel syndrome and diet. Gastroenterol. Rep. 2017, 5, 11-19. [CrossRef] [PubMed]

56. Arora, T.; Sharma, R. Fermentation potential of the gut microbiome: Implications for energy homeostasis and weight management. Nutr. Rev. 2011, 69, 99-106. [CrossRef]

57. Nicholson, J.K.; Holmes, E.; Kinross, J.; Burcelin, R.; Gibson, G.; Jia, W.; Pettersson, S. Host-gut microbiota metabolic interactions. Science 2012, 336, 1262-1267. [CrossRef] [PubMed]

58. Fukuda, S.; Toh, H.; Hase, K.; Oshima, K.; Nakanishi, Y.; Yoshimura, K.; Tobe, T.; Clarke, J.M.; Topping, D.L.; Suzuki, T.; et al. Bifidobacteria can protect from enteropathogenic infection through production of acetate. Nature 2011, 469, 543-547. [CrossRef] [PubMed]

59. Vernocchi, P.; Del Chierico, F.; Putignani, L. Gut microbiota profiling: Metabolomics based approach to unravel compounds affecting human health. Front. Microbiol. 2016, 7, 1144. [CrossRef]

60. Moens, F.; Van den Abbeele, P.; Basit, A.W.; Dodoo, C.; Chatterjee, R.; Smith, B.; Gaisford, S. A four-strain probiotic exerts positive immunomodulatory effects by enhancing colonic butyrate production in vitro. Int. J. Pharm. 2019, 555, 1-10. [CrossRef]

(C) 2019 by the authors. Licensee MDPI, Basel, Switzerland. This article is an open access article distributed under the terms and conditions of the Creative Commons Attribution (CC BY) license (http://creativecommons.org/licenses/by/4.0/). 